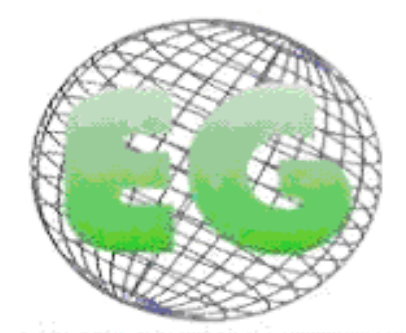

ISSN 1695-6141 N26

\title{
REVISIONES
}

\section{La investigación básica aplicada a la clínica en la lesión renal aguda}

A Pesquisa Básica aplicada à clínica na Lesão Renal Aguda

\section{*Barros de Moura Neiva, L., **Fernandes Vattimo, MDF.}

\author{
*Doctora en Enfermería. Programa de Salud del Adulto y Anciano. E-mail: lucianabmneiva@uol.com.br \\ **Profesora Asociada del Departamento de Enfermería en la Salud del Adulto. Escuela de Enfermería de la \\ Universidad de São Paulo. Brasil.
}

Palabras clave: Insuficiencia renal aguda; Iesión renal aguda; Investigación Básica; enfermería Palavras-chave: Insuficiência renal aguda; Lesão renal aguda; Pesquisa Básica; enfermagem Keywords: acute renal failure; acute renal injury; basic research; nurse

\section{RESUMEN}

Mucho se ha estudiado sobre la patogénesis de la insuficiencia renal aguda en modelos experimentales. Recientemente algunos autores consideraron que el término lesión renal aguda caracteriza de forma más completa esta condición. La utilización de cultura de células, tejidos, órganos y animales es esencial para reunir informaciones necesarias para el completo entendimiento de ese proceso mórbido. Se puede decir que las indagaciones se inician con el paciente, pero la comprensión de los mecanismos fisiológicos y patológicos necesita de modelos más simples.

Este estudio tiene por objetivo analizar el empleo de la investigación básica en la clínica en la lesión renal aguda y parte de la hipótesis de que el avance de la sociedad científica se basa en la aplicación de datos innovadores que posibiliten la definición de propiedad intelectual y que termine con la mejora de la salud de las poblaciones sin expectativa de aplicación inmediata de los resultados. La ciencia básica debe ser una experiencia de investigación que también compete al enfermero.

Se ha realizado una revisión bibliográfica en el período 1999-2009, en revistas indexadas y libros, las bases de datos Medline, LILACS, SciELO, en libros en lengua portuguesa e inglesa. Fue seguido por un análisis de los contenidos accesados que mejor se adecuaban al tema propuesto. Se utilizaron los siguientes términos: la lesión renal aguda, la investigación básica, la enfermería.

Se concluye que la investigación básica y la práctica clínica son complementarias y que deben llevarse a cabo por el equipo multidisciplinar. En este entorno, la enfermera se enfrenta con dos obstáculos: la dificultad en conocer los resultados de búsqueda y especialmente su aplicación. Es imperativo que los enfermeros se incluyan en el grupo que transmite la idea, pero también en el grupo que crea la idea. 


\section{RESUMO}

Muito tem sido estudado sobre a patogênese da insuficiência renal aguda em modelos experimentais. Mais recentemente alguns autores têm considerado que o termo lesão renal aguda (LRA) caracterize de forma mais completa esta condição, considerada a possibilidade de reversão. A utilização de cultura de células, tecidos, órgãos e animais é essencial para reunir informações necessárias para o completo entendimento desse processo mórbido. Os questionamentos iniciam sim com o paciente, mas, a compreensão dos mecanismos fisiológicos e patológicos necessita de modelos mais simples.

Este estudo tem por objetivo analisar o emprego da pesquisa básica na clínica da LRA e parte do pressuposto que o avanço da sociedade científica se pauta na aplicação de dados inovadores que possibilitem a definição de propriedade intelectual que culmine com a melhora da saúde das populações, sem a expectativa de aplicação imediata de resultados. A ciência básica deve ser mais uma experiência de pesquisa que também compete ao enfermeiro.

Nesse estudo foi realizada uma revisão bibliográfica no período de 1999 a 2009, em periódicos indexados nas bases de dados Medline, LILACS, SciELO, e em livros, em língua portuguesa e inglesa. Foi seguida então por uma análise dos conteúdos acessados que melhor se adequaram ao tema proposto. Foram utilizados os seguintes termos: lesão renal aguda, pesquisa básica, enfermagem.

Conclui-se que a pesquisa básica e a clínica se complementam e para isso devem ser realizadas por equipe multiprofissional. Neste ambiente, o enfermeiro se confronta com dois obstáculos: dificuldade em conhecer resultados de pesquisa e, principalmente a aplicação destes. É imperativo que o enfermeiro esteja inserido no grupo que transmite a idéia, mas também no grupo que cria a idéia..

\section{ABSTRACT}

There have been many studies on pathogenesis of acute renal failure in experimental models. More recently some authors have considered that the term acute renal injury characterizes this condition. The use of culture of cells, organs, tissues and animals is essential to gather necessary information for the complete understanding of this morbid process. The questions begin with the patient, but the understanding of the physiological and pathological mechanisms needs simpler models.

This study aims to analyze the use of the basic research in the clinical in the acute renal injury and it considers as true the hypothesis that the advance of the scientific society is based on the application of innovative data that make the creation of intellectual property possible and culminates with the improvement of the health of the populations, without the expectation of immediate applications of the results. Basic science must be one more experience of research that the nurses must take part in.

This study was a bibliographical research. Medline, LILACS and Scielo were accessed using "acute kidney injury, acute kidney failure, basic research and nursing" as descriptors. Studies in English and Portuguese language were taken. After that, the studies were analyzed.

The research concluded that basic and clinical research complement each other and should be performed by multiprofessional staff. It is important that nurses be the professional that sends the message, but also that hey need to create the message.

\section{INTRODUCCIÓN}

Durante la Segunda Guerra Mundial, la pérdida de la función renal fue descrita en víctimas de los incesantes bombardeos alemanes ${ }^{(1,2)}$. En la ciudad de Londres, individuos rescatados de los escombros se presentaban aparentemente sanos, con buena recuperación de las lesiones, mas con reducción del flujo urinario. Cuadro este después definido como insuficiencia renal aguda (IRA) ${ }^{(2)}$ relacionada con el trauma.

En aquel período, se utilizó el término necrosis tubular aguda debido a la evidencia histológica e incompleta de necrosis de túbulos renales en pacientes sometidos a autopsia. La mortalidad de la IRA se aproximó al 100\% en la II Guerra Mundial. En aquel momento, la 
hemodiálisis todavía no estaba disponible, lo que contribuyó a agravar el cuadro y el pronóstico de las víctimas del síndrome de aplastamiento (crush syndrome) ${ }^{(1,2)}$.

En la Guerra de Corea en 1950, militares heridos fueron sometidos a largas sesiones de hemodiálisis, lo que llevó a una reducción de la mortalidad de $90 \%$ a $50 \%{ }^{(1,2)}$. En la Guerra de Vietnam, otra técnica desarrollada ayudó a reducir la IRA, el "intracath", dispositivo de punción venosa profunda, que facilitó la reposición de grandes volúmenes a los soldados heridos en los campos de batalla ${ }^{(2)}$.

La evaluación de la incidencia de IRA en estos conflictos militares demostró cierta mejora. Algunos factores marcaron la diferencia, entre ellos la rapidez en la reposición de fluidos en los campos de batalla asociada a la evacuación también rápida de víctimas para los hospitales por medio de helicópteros ${ }^{(1)}$. El análisis de estos datos supuso que medidas rápidas, como las implementadas, podían prevenir la IRA ${ }^{(1)}$.

Mucho se ha estudiado sobre la patogénesis de la IRA en modelos experimentales, mas lo cierto es que la mortalidad actual permanece elevada, semejante a la II Guerra Mundial, principalmente en grupos específicos. Con todo, algunos factores clínicos han cambiado. El escenario actual compete a pacientes más ancianos y enfermedades crónicas con insuficiencias orgánicas múltiples cada vez más graves ${ }^{(2)}$.

\section{METODOLOGÍA}

Esta revisión consideró investigaciones cualitativas que comprendiesen resultados relativos a la investigación básica asociada a la práctica clínica en la lesión renal aguda. El objetivo, responder a las siguientes preguntas: "¿La investigación básica y la investigación clínica en la LRA se complementan?" y “¿Cómo el enfermero está inserto en este proceso?"

Es una investigación bibliográfica, o de fuentes secundarias, donde el tema de estudio fue abordado a partir de una consulta bibliográfica que comprendió revistas, libros, monografías, tesis, periódicos, entre otros. ${ }^{(2)}$ Según Marconi y Lakatos, la finalidad de estudios como este es colocar al investigador en contacto directo con todo contenido escrito, dicho o filmado sobre determinado asunto, incluyendo conferencias seguidas de debates que habían sido transcritas de alguna forma, ya publicadas, ya grabadas. ${ }^{(2)}$

Fue elaborado un análisis bibliográfico, abarcando artículos científicos nacionales, internacionales y libros. Para proceder a la busca de estas referencias, se utilizaron las siguientes palabras clave: lesión renal aguda, investigación básica y enfermero(a). La estrategia de busca fue elaborada con el objetivo de accesar materiales escritos en inglés y portugués. Se realizaron dos etapas respectivas: la primera consistió en la busca inicial de las palabras clave en las bases "Descriptores en Ciencias de la Salud" (DeCS) disponibilizadas por la Biblioteca Regional de Medicina (BIREME). Se seleccionaron las versiones en portugués e inglés de los términos identificados en el DeCS, pues esta base dispone de términos en estos dos idiomas; la segunda etapa comprendió el rastreo de las siguientes bases de datos: Literatura Latino-Americana y del Caribe en Ciencias de la Salud (LILACS), Scientific Electronic Library Online (SciELO); Medical Literature Online (Medline); National Center for Biotechnology Information (PubMed - NCBI) y Base de Datos de Enfermería (BDENF). Se eliminaron las investigaciones que no correspondían al objetivo de esta revisión, las que no presentaron datos cualitativos y las que estaban repetidas. 


\section{Desarrollo}

\section{Definición /Clasificación/ Terminología más actual}

La insuficiencia renal aguda presenta un concepto universal. Es un síndrome caracterizado por un deterioro abrupto de la función renal, con declive de la tasa de filtración glomerular, acompañada por un acúmulo de productos nitrogenados, como nitrógeno uréico sanguíneo y creatinina sérica, relacionado o no con la reducción del volumen urinario ${ }^{(3)}$.

Clasificada en tres subtipos, la forma más común es la IRA intrínseca. Representa el 35 a $40 \%$ de los casos ${ }^{(3,4)}$, estando causada principalmente por la isquemia. La segunda causa más frecuente son las nefrotoxinas, de ahí los términos IRA isquémica e IRA nefrotóxica ${ }^{(5)}$.

A pesar de muchas investigaciones y de la avanzada tecnología el pronóstico actual de la IRA no es estimulante. La definición de insuficiencia renal aguda es compleja pudiendo estar asociada a una necesidad de diálisis o a un leve aumento en la creatinina sérica ${ }^{(4)}$. Paralelamente, el empleo del término necrosis tubular aguda es paradójico dado que la constatación de necrosis en tejidos está casi siempre ausente, cuando no es heterogénea y difusa.

Algunos autores señalan que el término lesión renal aguda comprende de forma más completa esta condición. La lesión renal aguda puede ser relacionada con un problema fisiopatológico causado por múltiples factores, que ocurre en varios ambientes y con manifestaciones clínicas que surgen en cadena, desde discreta, más prolongada elevación del nivel de creatinina sérica hasta la anúria ${ }^{(4)}$.

La nueva definición de la IRA ha ganado énfasis y fue tema de un de los grupos de investigación de la Sociedad Americana de Nefrología (ASN). En el encuentro de la ASN en 2005, la terminología "acute kidney injury" o lesión renal aguda se estableció como preferida para esta alteración clínica ${ }^{(6)}$. Por tanto, a partir de este momento será utilizado el término lesión renal aguda.

\section{Morbidad y Mortalidad}

La epidemiología de la lesión renal aguda en pacientes críticamente enfermos en diversas regiones del mundo no presenta consenso ${ }^{(7)}$. Muchos estudios se rigieron por datos de un único centro, pero relacionado con un solo país ${ }^{(7)}$. El periodo de prevalencia y mortalidad registrados presentaban variaciones amplias y lo que más dificultaba la comparación entre los resultados obtenidos eran los variados criterios de inclusión ${ }^{(7)}$.

En 2005 se publicó el primero gran estudio de impacto de enfoque internacional sobre la epidemiología y los resultados de la lesión renal aguda en pacientes gravemente enfermos. Tal estudio se realizó en 54 centros en 23 países, entre ellos Brasil, con participación de cuatro centros. Dada la importancia del referido estudio y considerando la relevancia de la inserción del escenario nacional, tales resultados serán aquí expuestos ${ }^{(7)}$.

Los criterios para lesión renal aguda fueron oliguria, con volumen urinario inferior a $200 \mathrm{ml}$ en 12 horas y/o azotemia acentuada a partir de un nivel de nitrógeno ureico sanguíneo superior a $84 \mathrm{mg} / \mathrm{dl}$ ( $>30 \mathrm{mmol} / \mathrm{L})$. La intención de este estudio estudiar la LRA más severa, predominantemente aquellas tratadas con terapia de sustitución renal ${ }^{(7)}$.

A partir de un número aproximado a 30.000 pacientes, se verificó una prevalencia de LRA de 
$5,7 \%$, la mortalidad hospitalaria fue significativamente más alta que el índice de mortalidad (SAPS II), $60,3 \%$ versus $45,6 \%$. Se registraron $52 \%$ de óbitos en unidades de cuidados intensivos y $8 \%$ murieron en el hospital tras el alta de la $\mathrm{UCl}$. De los pacientes que recibieron alta hospitalaria, 13,8\% necesitaron de terapia de sustitución renal. La duración media de permanencia en la UCI fue de 10 días y en el hospital de 22 días. La causa más común para LRA fue choque séptico, con una frecuencia de $47,5 \%{ }^{(7)}$.

Es importante señalar que el periodo de prevalencia, el índice de mortalidad y la mortalidad hospitalaria en Brasil fue respectivamente 4,8\%, 43,6\% e $76,8 \%$, resultados muy preocupantes, no muy diferentes del episodio mundial.

Lo cierto es que estudios como estos, proporcionan además de datos estadísticos, nuevas posibilidades de intervención que contribuyen al pronóstico de pacientes con LRA.

\section{Mecanismos de la LRA}

A partir de los años 80 se tiene claro que al menos otros dos mecanismos, distintos de la necrosis, contribuyen al desarrollo de la NTA. Primero, la lesión subletal y reversible a las células tubulares renales ha sido reconocida como un factor importante que favorece la disfunción tubular de la LRA; segundo, está cada vez más claro que la apoptosis, una forma de muerte celular auto inducida, también contribuye a las formas isquémica y nefrotóxica de la LRA.

El riñón es susceptible al ataque nefrotóxico por dos factores: el volumen sanguíneo que recibe, en torno al $25 \%$ del débito cardiaco y por su capacidad de concentrar elevados niveles de toxinas en el intersticio medular y en las células epiteliales renales ${ }^{(2,3)}$.

La reducción del ritmo de filtración glomerular puede estar provocada por factores que pueden llevar a vasoconstricción renal y consecuente disminución del flujo sanguíneo renal. Otros agentes pueden lesionar el túbulo renal por acción tóxica, provocando efectos similares a los mencionados para la isquemia ${ }^{(2)}$.

Esta descripción resumida de las alteraciones estructurales y funcionales que caracterizan la fisiopatología de la LRA es resultado de estudios desarrollados en bancos de laboratorios, muchos con modelos animales, y seguramente nos ayuda a vislumbrar mayores posibilidades de intervenciones a nivel molecular y celular para la reversibilidad de esta enfermedad renal.

A continuación se citan algunos ejemplos del impacto de la investigación básica en la salud de las poblaciones. Algunas de estas investigaciones presentan resultados aparentemente accidentales.

\section{Penicilina e Insulina}

La Penicilina fue descubierta en 1928 por Alexander Flemming, en St Mary en Londres. En 1940 fue purificada por Howard Florey y Ernst Chain, ambos de la Universidad de Oxford ${ }^{(10)}$. Sus experimentos con ratones infectados comprobaron su acción antibiótica y su eficacia y aparente ausencia de toxicidad.

A partir de 1941 comenzaran tests humanos. El primer hombre tratado con penicilina fue un agente de polícia que sufría de septicemia por abscesos diseminados. Su mejora fue sorprendente; con todo, cuando las reservas del antibiótico se agotaron este falleció ${ }^{(10)}$. 
Hasta casi 20 años después de los primeros tests la penicilina no llegó al público ni tuvo la representatividad terapéutica incuestionable de hoy.

El caso de la insulina caracteriza otro ejemplo, aunque distinto, de la aplicación de la investigación básica en la clínica. En 1905, Paul Langerhans al estudiar el páncreas a través de um microscopio encontró células totalmente desconocidas en el tejido exocrino (islotes de Langerhans). Posteriormente, Edouard Laguesse vio que tales células tenían relación con algún tipo de secreción que participara en el proceso de digestión ${ }^{(11)}$.

En 1889, Oscar Minkowski y Joseph von Mehring extrajeron el páncreas de un perro sano para demostrar el papel del órgano en la digestión de alimentos confirmando la relación entre el páncreas y la Diabetes. A prinicipios del siglo, Eugene Opie consiguió relacionar los islotes de Langerhans y la Diabetes ${ }^{(11)}$.

En los 20 años siguientes investigadores insistieron en el aislamiento de la secreción de los islotes a partir de modelos animales con perros y becerros. En 1922 se administró la primera inyección de insulina en un ser humano con resultados benéficos, resultado de manipulaciones de ingeniería genética, disciplina esta muchas veces desconocida por investigadores clínicos ${ }^{(11)}$. Inútil aquí destacar la obviedad de la importancia clínica actual de la insulina.

\section{Modelos experimentales y Clínicos}

El gran triunfo de la investigación dirigida a la enfermedad está relacionado con la mejora de cuidados al paciente y la reducción de la morbilidad y mortalidad. Los interrogantes se inician con el paciente, mas la comprensión de los mecanismos fisiológicos y patológicos necesita de modelos más simples, libre de variables muchas veces incontrolables ${ }^{(12)}$.

La utilización de cultivo de células, tejidos, órganos y animales y la reconstitución de modelos es primordial para la obtención de informaciones necesarias para el completo entendimiento del proceso de la enfermedad. La ciencia básica en biología molecular y celular, además de los usuales modelos "in vivo", son fundamentales para la evolución del conocimiento de la fisiopatología de la LRA $^{(12)}$.

El uso de modelos más simples, así como sus informaciones, necesita de la corroboración en modelos intactos. Informaciones "in vitro" deben ser también consistentes en modelos "in vivo" antes de seguir con estudios más extensivos ${ }^{(12)}$.

En cuanto a la lesión renal aguda, un nuevo concepto proporcionó un mayor entendimiento de la fisiopatología de la categoría isquémica: la lesión reversible y subletal a las células tubulares renales contribuye fuertemente a la disfunción tubular renal, o sea, la disfunción clínica con retención hídrica y escorias no tiene relación tan estrecha como se imaginaba con la lesión celular por necrosis ${ }^{(12)}$.

Reconocer que la lesión subletal favorece la disfunción tubular vista de la LRA clínica representa ejemplo consistente de cómo el intercambio de ideas entre investigadores del área clínica y básica, así como la utilización de un número de modelos diferentes de investigación, pueden complementarse uno con el otro en la comprensión de la enfermedad humana ${ }^{(12)}$.

Es sabido que la lesión tubular lleva a la reducción de la tasa de filtración glomerular por 
medio de la retrodifusión del filtrado glomerular para el intersticio renal y sistema venoso renal. Este mecanismo disfuncional se mostró inicialmente en animales en la década de los 70. Posteriormente, pacientes con NTA fueron sometidos a la misma investigación de forma positiva, confirmando que la retrodifusión es factor relevante para la disfunción renal en la NTA isquémica. Resultados como estos demuestran que ideas generadas en laboratorio pueden ser confirmadas en humanos ${ }^{(12)}$.

Algunos ejemplos de resultados de investigación experimental y clínica desarrollados en la Universidad de São Paulo serán citados. Watanabe sometió ratones a una situación de isquemia constatando LRA no oligúrica por la alteración de los criterios de evaluación de la función renal, como depuración de creatinina y actividad de enzima antioxidante El pretratamiento con isoflavona, un fitoquímico encontrado en el grano de soja, asociado al hemin (agente químico inductor de la enzima $\mathrm{HO}-1$ ) mostró mejora significativa de la función renal en este modelo isquémico. La isoflavona es reconocida por sus propiedades antioxidantes y antiaterosclerótica $^{(13)}$.

Shibuya sugirió en un mismo modelo isquémico que el efecto antioxidante de la estatina proporcionó protección a la función renal, avalada or la depuración de creatinina, peróxido urinario y osmolaridad urinaria. La estatina es una droga con acción antiinflamatoria que reduce niveles de colesterol, siendo administrada en pacientes dislipidémicos ${ }^{(14)}$.

Venkatachalam afirmó que la utilización de modelos experimentales para estudiar la LRA nefrotóxica ha encontrado algunos obstáculos. La administración de aminoglicosídeos, radiocontrastes, o endotoxinas, en ausencia de otros ataques renales paralelos, induce LRA en ratones, mas tal resultado no es fiable. Investigadores han demostrado la importancia de combinar ataques para imitar la NTA en humanos. Otro estudio constató que la nefrotoxicidad de la gentamicina fue más acentuada en un medio donde se identificó bacteremia gram-negativa asociada ${ }^{(12)}$.

Por otro lado, Hosaka demostró que el tratamiento con gentamicina en ratones determinó lesión renal aguda no oligúrica. A piora de la función renal se observó cuando la gentamicina fue asociada a un antiinflamatorio no hormonal, la indometacina. Inversamente, no se observó potencial nefrotóxico en el tratamiento gentamicina cuando asociada a rofecoxibe, un AINH selectivo de COX-2, actualmente no disponible para uso clínico ${ }^{(15)}$.

Estudios han demostrado también que la administración de contraste radiológico produce lesión en ratones asociada a otros estresantes renales como agotamiento de volumen e inhibición de la producción de prostaglandina ${ }^{(12)}$.

En trabajo reciente, Ferreira Pinto confirmó lesión renal aguda no oligúrica en ratones sometidos a contrastes yodados, sus datos sugieren que el uso de la n-acetilcisteína aislada, medicamento con efecto antioxidante, tiene efecto protector sobre el riñón, atenuando la toxicidad del contraste. Su asociación con hiperhidratación, medida frecuentemente empleada en humanos, no produjo mejora adicional. Tales tratamientos, cuando separados, confirieron protección ${ }^{(16)}$.

En otro modelo de nefrotoxicidad "in vitro", Neiva verificó que la toxicidad de la polimixina B en células tubulares renales dependió de la dosis y tiempo, o sea, fue mayor en la medida que los dos parámetros aumentaban, este efecto nefrotóxico se atenuó por el hemin, inductor químico de una enzima $(\mathrm{HO}-1)^{(17)}$. Datos sobre la nefrotoxicidad de la polimixina $\mathrm{B}$ en humanos han sido validados y demostrado su toxicidad. 


\section{Investigación Clínica y Básica: ¿cómo se complementan?}

Molitoris propone un ciclo continuo que debería existir entre conceptos elaborados y tests de estas hipótesis a partir de modelos experimentales, sería una comparación de poder y fragilidad de los modelos disponibles de LRA utilizando una escala relativa. Este investigador reconoce que el grado de complejidad en los humanos es mucho más fuerte así como sus limitaciones experimentales. Por otro lado, la posibilidad de manipulación, de aislar variables y la comprensión de los mecanismos son mayores en modelos con cultivo de células. Inversamente, el valor terapéutico es mayor en modelos animales y humanos ${ }^{(12)}$.

En trabajo clínico orientado por el LEMA, Magro verificó la frecuencia de LRA en el posoperatorio de Revascularización del Miocardio con y sin circulación extracorpórea. Entre los resultados, se registró que la cirugía de $\mathrm{RM}$ asociada a otro abordaje quirúrgico no reveló mayor predisposición a la ocurrencia de LRA. Además, la depuración de creatinina y la urea mostraron ser factores predictivos para LRA en cuanto que la fracción de excreción de sodio (FeNa) y la creatinina urinaria mostraron ser factores diagnósticos ${ }^{(18)}$.

En trabajo más reciente, Magro clasificó la función renal de pacientes en el pos-operatorio de cirugía cardiaca utilizando el sistema clasificador RIFLE además de haber evaluado la Cistatina C, un marcador de tasa de filtración glomerular. La LRA ocurrió en 78,5\% por el criterio Rifle. La Cistatina C presentó mayor sensibilidad como marcador de LRA que la creatinina plasmática ${ }^{(19)}$.

Ya Mizoi al evaluar la relación entre la creatinina plasmática y proteína carreadora de retinol (RBPu), dos marcadores de LRA, verificó que pacientes con mayores valores de RBPu tenían solos cuatro posibilidades de presentar creatinina normal, o sea, la sensibilidad de $\mathrm{RBPu}$ como test diagnóstico todavía es cuestionable ${ }^{(20)}$.

Tales estudios clínicos destacan la necesidad de tratamientos profilácticos así como la inserción de marcadores precisos y sensibles para garantizar un diagnóstico precoz y seguro de LRA.

La continuidad del progreso en los cuidados al paciente depende de la relación entre investigadores del área básica y clínica. La asociación de hipótesis obtenidas a partir de modelos experimentales y el test de estas hipótesis en humanos son la estrategia más exitosa para el progreso en el área ${ }^{(12)}$.

\section{CONSIDERACIONES FINALES}

La investigación básica es realizada por un equipo multiprofesional y el enfermero está comenzando a destacar en este ambiente. Los enfermeros tienen dos obstáculos: dificultad en conocer los resultados de investigación y la aplicación de los mismos. Todo ello determinado por dificultades de diferente origen ${ }^{(21)}$.

El conocimiento tiene que ser diseminado, mas también actualizado. El enfermero puede estar incluido en el grupo que transmite la idea, mas también en el grupo que crea la idea. Es preciso resaltar que el avance de una sociedad se basa en la implementación de datos innovadores que posibiliten la mejora de la salud, sea física, mental o espiritual.

Destaca en este contexto la afirmación oportuna de Angerami: "en esta década, precursora del siglo XXI, será fundamental dar continuidad a la preparación de investigadores y profundizar en los estudios que permitan evaluar la calidad de las investigaciones realizadas. 
La escasez de recursos a nivel mundial será un factor selectivo para proyectos. Solamente serán reconocidos aquellos con calidad e impacto en la práctica, lo que nos lleva a repensar nuestras líneas de investigación, nuestros estudios para publicación y la calidad de los periódicos donde estamos divulgando el conocimiento producido. No basta producir, la fase de aprendizaje ya fue superada, el investigador del próximo siglo deberá ser exigente con su producción, pertenecer a grupos de investigación que favorezcan la discusión y la aparición de nuevas ideas que fortalezcan el debate y la multidisciplinaridad, sólo así tendrán el reconocimiento de la comunidad científica. El proyecto calidad llegó también para la investigación en Enfermería. Es preciso osar, prever, experimentar, evaluar, participar de los anhelos de las poblaciones, e introducir nuevas prácticas que ofrezcan soluciones ${ }^{(22) . "}$

\section{LEMA}

El Grupo de Investigación LEMA (laboratorio experimental de modelos animales) se inició en 1998, fue cadastrado en el CNPq em 1999, estando compuesto actualmente por nueve investigadores del área de enfermería. Produce trabajos de iniciación científica, mestrado y doctorado. Su investigación está orientada a la lesión renal aguda, donde la gran mayoría de los trabajos incluye la investigación básica "in vivo" e "in vitro", realizándose también trabajos en el área clínica. Su sede se localiza en la Escuela de Enfermería de la Universidad de São Paulo, teniendo como orientadora a la Profa. Dra. Maria de Fátima Fernandes Vattimo.

\section{REFERÊNCIAS}

(1) Schrier RW, Wang W, Poole B, Mitra A. Acute renal failure: definitions, diagnosis, and therapy. J Clin Invest 2004; 114(1): 5-14.

(2) Yu L, Burdmann E, Seguro AC, Helou CMB, Zatz R. Insuficiência renal aguda. In: Zatz R, editor. Fisiopatologia renal. São Paulo: Atheneu; 2000.cap.14, p.261-97.

(3) Brady HR, Brenner BM, clarkson MR, Lieberthal W. Acute renal failure. In: Brenner BM, Rector FC Jr, editors. The kidney. 6 th ed. Philadelphia: WB Saunders; 2000.v.1, cap.28; 18(5): 505-18.

(4) Devarajan P. Update mechanisms of ischemic acute kidney injury. J Am Soc Nephrol 2006; 17: 1503-520.

(5) Boim MA, Santos OFP, Schor N. Insuficiência renal aguda: etiologia diagnóstico e tratamento. In: Schor N, Srougi M, editores. Nefrologia, urologia, clínica. São Paulo: Sarvier; 1998.cap.8, p.20-3.

(6) Warnock DG. Towards a definition and classification of acute kidney injury. J Am Soc Nephrol 2005; 16:3149-150.

(7) Uchino S, Kellum JA, Bellomo R, Doig GS, Morimatsu H, Morgera S, et al. Acute renal failure in critically ill patients: A multinational, multicenter study. JAMA 2005; 294 (7): 813-818.

(8) Lieberthal W. Biology of acute renal failure: therapeutic implications. Kidney Int 1997; 52 (4): 1102-115.

(9) Cronin RE, Henrich WL. Toxic nephropaties. In: Brenner BM, Rector FC Jr, editors. The kidney. $6^{\text {th }}$ ed. Philadelphia: WB Saunders; 2000. v.1, cap.34, p.1563-96.

(10)Mandell GL, Petri WA Jr. Fármacos antimicrobianos. Penicilinas, cefalosporinas e outros antibióticos $\square$-lactâmicos. In: Goodman LS, Gilman AG. As bases farmacológicas da terapêutica. 9a ed. Rio de Janeiro: McGraw-Hill Interamericana; 1996. p. 790-811.

(11)Davis SN, Granner DK. Insulina, fármacos hipoglicemiantes orais e a farmacologia do pâncreas endócrino. In: Goodman LS, Gilman AG. As bases farmacológicas da terapêutica. 9a ed. Rio de Janeiro: McGraw-Hill Interamericana; 1996. p. 1103-25.

(12)Lieberthal W, Nigam SK. Acute renal failure II. Experimental models of acute renal failure: imperfect but indispensable. Am J Physiol Renal Physiol 2000; 278: F1-F12.

(13)Watanabe M. A isoflavona e o sistema heme-oxigenase na insuficiência renal aguda 
isquêmica em ratos.[dissertação] São Paulo (SP): Escola de Enfermagem da Universidade de São Paulo; 2005.

(14)Shibuya CA. Papel da heme-oxigenase na proteção pelas estatinas na insuficiência renal aguda isquêmica em ratos. [dissertação] São Paulo (SP): Escola de Enfermagem da Universidade de São Paulo; 2006.

(15)Hosaka EM, Santos OFP, Vattimo MFF. Effect of cyclooxygenase inhibitors gentamicininduced nephrotoxicity rats.. Braz J Med Biol Res 2004; 37: 979-85.

(16)Pinto CF. Hidratação e n-acetilcisteína na insuficiência renal aguda por contraste iodado: estudo experimental em ratos [dissertação] São Paulo (SP): Escola de Enfermagem da Universidade de São Paulo; 2005.

(17)Neiva LBM. O papel da heme oxigenase na lesão celular desencadeada pelo sulfato de polimixina B em células LLCPK1. [dissertação] São Paulo (SP): Escola de Enfermagem da Universidade de São Paulo; 2004.

(18)Magro MCS, Vattimo MFF. Does urinalysis predict acute renal failure after heart surgery? Ren Fail 2004; 26: 385-92.

(19)Magro MCS. Cistatina C e RIFLE: avanços na avaliação da função renal em pós-operatório de cirurgia cardíaca. [Tese] São Paulo (SP): Escola de Enfermagem da Universidade de São Paulo; 2007.

(20)Hokama CSM. Função renal de pacientes de UTI: creatinina plasmática e proteína carreadora do retinol urinário (RBPu).[dissertação] São Paulo (SP): Escola de Enfermagem da Universidade de São Paulo; 2004.

(21)Angerami ELS. O desenvolvimento da pesquisa no Brasil. Rev Latino-Am. Enfermagem 1993; 1:43-52.

(22) Marconi MA, Lakatos EM, Fundamentos da Metodologia Científica. $6^{\circ}$ Ed. São Paulo: Atlas; 2005. 